Aleksander Gemel

PAULA QUINON

\title{
THE APPROXIMATE NUMBERS SYSTEM AND THE TREATMENT OF VAGUENESS IN CONCEPTUAL SPACES ${ }^{1}$
}

\section{Introduction}

The theory of conceptual spaces is intended to provide a framework for models of both symbolic and non-symbolic representations of knowledge and information (Gärdenfors 2000). As such, it seems to us to be very clearly suited to modeling pre-verbal representations belonging to a so-called core cognition, whose existence is postulated by cognitive developmental psychologists (Feigenson et al., 2004; Carey, 2009). In this paper we propose a treatment of representations of quantity that embraces both symbol-based and pre-verbal numerical concepts.

The representations we aim to study are related to the Approximate Number System (ANS). Cognitive psychologists claim that humans share with animals an abstract sense of quantity: they have a so-called "number sense". To "number sense" amounts two core systems of representations, which get activated by different core mechanisms: the ANS is one of these mechanisms (Dehaene 1997, 2008; Gallistel 1993; Feigenson et al., 2004; Carey, Sarnecka, 2006).

The ANS is a core system in the sense that it is present in human apprehension of quantities before verbal conceptual these of quantities appears. But, it is

1 The core idea of this paper was first formulated in a private conversation of the second author (the authors' names are listed in alphabetical order) with Jakub Szymanik in the summer 2010. The research of the second author has been supported by the IEF FP7 Marie Curie Fellowship "Numbers" (PIEF-GA-2011-301470). We wish to express our gratitude to Peter Gärdenfors for comments on an earlier version of this paper. 
claimed, it also underlies symbolic number-concept creation and it is active in the processing of quantitative information for the entire life of an individual. Firstly it reacts to nonverbal input and this remains the case through the whole lifespan (Halberda, Feigenson, 2008), it also gets activated through symbolic input. To exemplify the functioning of the ANS and the ANS-related representations, let us consider the following case: the reaction to a set of 17 elements corresponds to the seventeenth element of the ANS-sequence, i.e. a set containing 17 with some specific neighboring area.

It is not our objective to postulate anything about the relation between exact number names, i.e. numerals, and representations related to the ANS (Halberda, Feigneson, 2008; Mussolin et al., 2012; Wagner, Johnson, 2011; see also Nagen, Sarnecka, 2015). Instead, we observe that numerals are in certain contexts interpreted as vague terms (Krifka, 2009; Solt, 2011). It might seem surprising, as at first glance there is nothing less vague than a number. "Seven" refers to seven; "twelve dwarfs" are twelve entities of the type "dwarf", etc. Naturally, there are some numerical expressions that embrace vagueness, like expressions consisting of a numeral and a modifier (e.g., "roughly ten", "more or less a hundred", or "about twenty") or generalized numerical quantifiers (e.g., "more" or "little", when applied to discrete sets of entities, etc.); but vagueness is usually not attributed to exact number names (i.e. numerals). Nevertheless, when the use of language is studied more carefully, one can see that in some contexts numerals behave like vague terms. This is the case, for example, in expressions like "there are 100 people in the room" or "two thousand people participated in the demonstration", in which case the intention of the speaker is to approximate the number of people (Dehaene, 1997, 2008).

Our aim in this paper is to conduct a preliminary study of a possible correlation between three concepts: exact numerals, in particular when used as vague quantifiers, ANS-related representations as a (naturalized) semantics for such expressions, and a model of the ANS-related representations in the framework of conceptual spaces. Our study amounts to an overall understanding of the structure of the ANS.

It should be clear from the beginning that we work under several arbitrary assumptions. First, we work in a paradigm in which "number sense" exists. Second, we are primarily interested in theoretical investigation and no experimental study has been proposed to verify whether there really exists a correlation 
between ANS-related representations and symbolic representations of exact numerals used as vague quantifiers. Conducting such a study would be very valuable not only for the current endeavour; it would also shed additional light on the nature of the ANS.

\section{Two core systems of numerical representation and exact numerals}

According to a paradigm defended by many cognitive scientists (Spelke, et al., 2007; Carey, 2009), at the basis of human numerical abilities lay two innate core cognitive systems responsible for pre-verbal representations of quantitative content, which in some way support interpretation of numerical expressions when the symbolic concept of a number is created: the system of parallel individuation (which allows identification in an exact way of sets up to four elements); and the system of approximate numbers (which allows approximation of cardinality of sets bigger than five elements) (Feigenson et al., 2004).

\subsection{Approximate Number System}

The system of approximate numbers (ANS) is a cognitive system that provides a mental representation of the approximate quantity of elements in the set. The size of the ANS-related representation is proportional to the number of perceived units and for this reason ANS-related representations are often referred to as analogue magnitude representations (ANRs). The analogue characteristic of the ANS means that all input provided by the system are represented by continuous data. In other words, a mental representation of a set consisting of 20 elements will be about twice as large as the mental representation of a set consisting of 10 elements.

The ANS-related representations work as an interconnected whole: an approximation of the cardinality of a given set is possible because it is put into correspondence with other representations. All estimations of size happen through comparison to the rest of elements. Comparisons of sizes of multiple sets are based on the function of their ratio. In other words, the distinction between 
a 10-element set and a 20 -element set is as difficult to make as the distinction between 20 - and 40 -element sets, since in both cases the ratio of the amount of elements in the sets is 1:2. The straightforward consequence of this observation is that the difficulty of discriminating between two sets is not dependent on absolute difference between their cardinalities. This seems quite intuitive when we analyze a case in which the absolute difference is the same. Let us take two pairs of sets: the first pair consisting of a 10-element set and a 20 -element set, and the other consisting of a 200-element set and a 210-element set. Although the absolute difference between the amounts of the elements of two sets is 10 , it is intuitively correct, and also experimentally proven, that it is much easier to distinguish between the first pair of sets than the second.

Two effects describe behaviour of the ANS:

1) the size effect, when the absolute difference between the two pairs of sets is constant, then sets with a smaller number of elements are easier to discriminate. This relation is illustrated by the example described above;

2 ) the distance effect, according to which it is easier to discriminate sets that differ by a significantly large number of elements. For instance, it is easier to notice the difference between 20 and 35 elements in the set then between 20 and 23 elements in the set.

\subsection{Parallel Individuation System}

The Parallel Individuation System (PIS) is not a quantity-specific, since it is not directly intended to represent numbers. Instead it is intended to create and sustain, in working memory, mental models consisting of a small number of objects - such as things, sounds, events, etc. The small number of individuals constituting the mental representation is a result of human cognitive limitations: in particular, the capacity of the working memory is usually limited to 4 objects at a time. Unlike the ANS, which is responsible for analogue representations of approximate cardinalities of sets as continuous wholes, the PIS is responsible for the representation of separate individuals. That is, the parallel individuation system provides no common symbol for "two" or "three" elements, but offers two or three separate symbols for each element respectively (each of the separate symbols may also carry information concerning its properties, or specifying its type). 


\subsection{Linguistic Number System}

None of the presented above innate cognitive systems can provide representations of the exact-number concept. The PIS provides representations only for up to four elements, and is not quantity-specific. The ANS accounts for approximate values. Cognitive scientists claim that exact numbers representations appear in consequence of functioning of the symbolic cognitive system: the Linguistic Number System (LNS). This system consists of numerals and also of various types of quantitative quantifiers. Symbols get their interpretation through the core cognitive systems, but also through interactions with other concepts in the process called conceptual "bootstrapping".

\section{The ANS and vagueness}

An inherent feature of the ANS is the approximate or vague character of both the inputs it processes and the representations these inputs activate. $\mathrm{Nu}$ meral expressions that first come to mind in relation to the ANS are generalized quantifiers such as "many", "little", "more”, "less", or "equally"; or numerical quantifiers used with a modifiers such as "roughly 20 " or "about 1000 ". In some contexts exact numerals are also used to approximate quantities. This paper is principally devoted to the third case. A prototypical example of use of an exact numeral to approximate a quantity is, for instance, when one says "this morning there were 100 people in the lecture hall" in order to estimate the number of people participating in some gathering.

In this paper we assume that the correct semantics for exact natural numbers, especially when used as vague quantifiers, is the ANS-related system of representations. It seems plausible since, as it is claimed by cognitive scientists, it is exactly this system of representations that gets activated by any quantitative input asserted without counting elements. This means that exact numbers used in a specific context and for a specific purpose (i.e. to estimate cardinalities bigger than four) may be considered vague terms. For instance, when one says "there are 100 people in the room" or "there are 1000 leaves on the tree", or "4000 people participated in the event", the objective of the speaker clearly does not consist in providing the exact cardinality of the set, but just in approximating it. 


\subsection{Vagueness}

Traditionally, the concept of vagueness relates to terms. A vague term is a term that has borderline cases of interpretation: when we say that the term "red" is vague it means that there are cases of redness that are difficult for a human to classify as red. In other words the semantics for vague terms is characterized by the existence of so-called borderline cases. According to Shapiro, who adopts the definition given by McGee and McLaughlin, an "object $a$ is the borderline case of predicate $F$, if $F a$ is 'unsettled' i.e., if $a$ is not determinately an $F$, nor is $a$ determinately non- $F$ " (Shapiro, 2006: 7). The technical term "determinately", used in the above definition, was introduce by McGee and McLaughlin as follows: "to say that an object $a$ is determinately an $F$ means that the thoughts and practices of speakers of the language determine conditions of application for $F$, and the facts about $a$ determine that these conditions are met" (McGee, McLaughlin, 1994).

Besides vague terms related to a quality (ex. bald, old), there are numerical quantifiers that are vague ("many", "little", "more", "less", "about 100"). Moreover, and this is what we study in this paper, even exact numerals, when used in specific contexts, can also be considered vague terms. Strictly speaking we are interested in understanding semantics underlying exact numerals when these are used to estimate cardinalities bigger than four, and behave as vague expressions.

As we mentioned above, a term is vague if it has borderline cases of interpretation, and some object can be called a borderline case of some predicate if the thoughts and practices of speakers of the language don't determine the perfect conditions of application for this predicate, and if facts about this object do not determine whether these conditions are met. Thus, in the context of the relationship between the ANS and vagueness, the question is whether the phrase "one hundred", used to estimate the approximate number of leaves on the tree, has borderline cases of interpretation. It seems obvious that the answer to this question is yes, since there are senses of " 100 " that are difficult for a human to classify as 100 when she is using the ANS. For instance, if someone asks a person to estimate the number of leaves on two trees, consisting of 100 leaves on the first tree and 103 leaves on the second, the answer would be probably " 100 " for both. The numeral " 100 " is used as a vague quantifier and it is so independently of speaker's intentions (whether she aimed at providing precise information or whether she just wanted to approximate).

Observe that since the representations provided by the ANS are vague, the size of the representation of the number 3 can't be exactly half as long as the rep-

\section{O) COGNITIVE SCIENCE}


resentation of number 6. Moreover, the vague nature of the ANS means that the boundaries of representations are blurred, which entails the possibility of confusion between two adjacent numerical representations. This phenomenon is in line with the characteristics of ANS size and distance effects. As demonstrated by numerous studies of the ANS, these effects are reflected in the difficulty of distinguishing, for example, 20- and 21-element sets from each other. Therefore, the first difficulty in modeling an ANS-related representation system in conceptual space consists in the necessity of considering the blurred nature of the boundaries of these representations. As we discussed above, the effect of blurred boundaries on the conceptual spaces model is specific to models of vague terms, where one uses clusters of prototypes.

\subsection{Spatial arrangement of ANS numerical representations}

Due to the analogue nature of the ANS, its numerical representations do reflect quantitative intuitions in such a way that they are best described with spatial categories. The type of representations that ANS generates is called analogue magnitude representations (AMRs). ${ }^{2}$ They are analogue because the neural entities that they activate are direct analogues of the magnitudes they represent. The particular type of AMRs that we are modeling activates in reaction to discrete quantitative magnitudes.

In the construction of the model, we use the idea that ANS-related representations are best characterized by spatial dimensions (Dehaene 1997, 2008; Carey, Sarnecka 2006). Thus the natural tool for modeling the representational structure of ANS-magnitudes appears to be the geometric representation model proposed by Peter Gärdenfors, known as the theory of conceptual spaces (Gärdenfors 2000).

\section{Conceptual spaces}

The main thesis of the theory of conceptual spaces developed by Gärdenfors is that the meanings of words can be represented as an organized spatial geometrical structure. Such a structure supports representation of many words

2 Beck (2014) provides a philosophical introduction to AMRs. 
simultaneously and provides a geometrical model for language representation. Concepts are represented as regions containing points in a one-dimensional or multidimensional space. Objects belonging to the extension of reference of the term being modeled are mapped onto these points. There is a metric (or a distance function) within the spaces. The distance between points expressed by the metric corresponds to some kind of similarity between the qualities of the points.

In the most general terms a metric is defined in the following way:

Definition: ${ }^{3}$ Consider a space $S$. A function $f$ defined on a space $S$ is a metric for $S$ iff for all points $a, b, c \in S$ :

(i) $f(a, b) \geq 0$, with $f(a, b)=0$ iff $a=b$;

(ii) $f(a, b)=f(b, a)$;

(iii) $f(a, b) \leq f(a, c)+f(c, b)$.

The metric is used to measure a degree of similarity between two points in the conceptual space. Quality dimensions, which can be taken into account, are of different types. They can be one of the three ordinary spatial dimensions - height, width, and depth - or some other physically measurable quality such as temperature, weight, brightness, or pitch. Finally they can have an abstract non-sensory character. The closer the two points are to one another, the more similar they are: if point $x$ is closer to point $y$ than to point $z$, then $x$ is more similar to $y$ than to $z$. In other words, the closer that representations of two points are placed in the space, the more similar are the objects represented by these points. Similarity can hence be defined as a monotonically decreasing function of the distance expressed by a metric, defined on the space.

Finally, concepts are represented as regions of conceptual space (sets of points). For example, red is a certain region in color space. But there is an important condition: not just any set of points in a space is a region of conceptual space in natural language, but only those regions that tend to have a specific topological feature. Namely, the concepts and properties have to correspond to convex regions in the given space. Convexity is defined as follows:

Definition: A region $R$ is convex iff for any two points $x$ and $y$ in $R$, all points between $x$ and $y$ are also in $R$.

3 This very general definition of metric is satisfied by many different metrics used in mathematics, for instance by the Euclidean metric. For our current endeavour particular differences between metrics are unimportant and hence will be ignored. 
This means that, as long as we consider a domain containing objects $x$ and $y$ as having some property, any object that is located between $x$ and $y$ will also have this property. For a convex region, one can describe positions within it as being more or less central.

Decisions about which points belong to one region are made on the basis of similarity to a distinguished point representing the prototypical example of the reference. One of the hypotheses of the theory of conceptual spaces is that (for many predicates) there exist prototypical examples of their reference (the prototype effect) (Gärdenfors 2000).

Mathematical counterparts of conceptual spaces are Voronoi diagrams (or tessellations), which exemplify a technique of dividing metrical space into cells. Every cell has a center, called a seed or generator, and contains all and only those points that lie closer to the given seed than to any other. Seeds model prototypes. In other words, a set of prototypes $\mathrm{P}=\left\{p_{1}, \ldots, p_{n}\right\}$ generates a set of convex regions $\mathrm{C}=\left\{c_{1}, \ldots, c_{n}\right\}$. Figure 1 is an example of this:

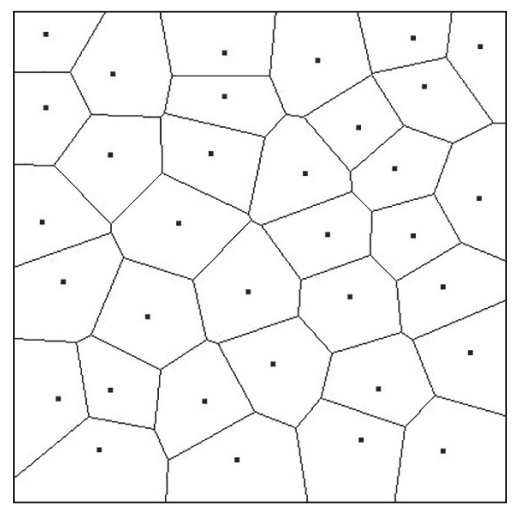

Fig. 1. A Voronoi tessellation

In the case of certain terms it can happen that it is more adequate to consider a set $\mathrm{P}^{*}$ of clusters of points $\left\{\left\{p 1_{1}, \ldots, p 1_{n}\right\},\left\{p 2_{1}, \ldots, p 2_{n}\right\}, \ldots,\left\{p n_{1}, \ldots, p n_{n}\right\}\right\}$ instead of a set P of points. As claimed by Decock, Douven (2014), see also Douven et al. (2013), this would be the case for vague concepts. Decock, Douven (2014) use the standard example of colors as requiring this cluster modeling: color names are vague, and modeling conceptual space with clusters of prototypes is specific to vague concepts. In other words, the meaning of some terms is more adequately modeled when one considers the division of the corresponding 
conceptual space as generated not by single points but by groups of points corresponding to prototypical representations of meaning. ${ }^{4}$

It is traditionally assumed that when the partition of a space is generated by a set $\mathrm{P}^{*}$ of clusters of points, these clusters form circles. That is, prototypical areas are circular, as shown in fig. 2.

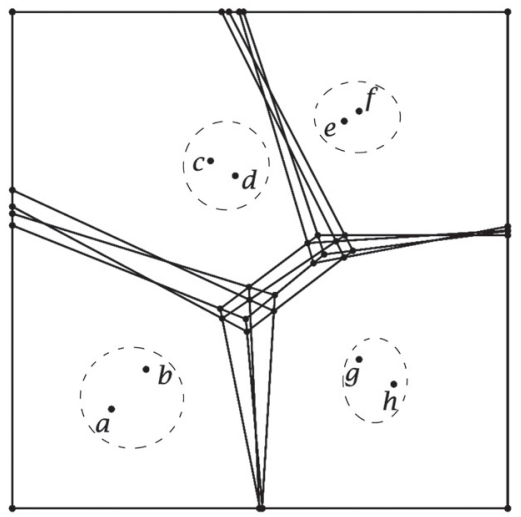

Fig. 2. Voronoi Diagrams generated by $\mathrm{P}^{*}$

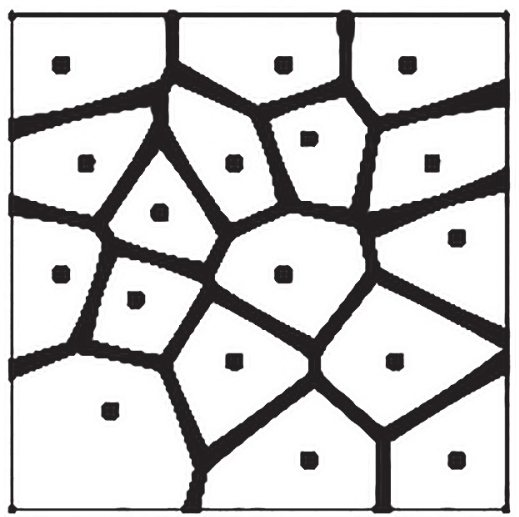

Fig. 3. Use of $\mathrm{P}^{*}$ to model vague concepts (Decock, Douven, 2014)

The tessellation made by the cluster of points formed in a circle (so-called vague prototypes), allows blurring of boundaries between concepts in the space.

${ }^{4}$ How to think about diagrams in this setting is presented in Gärdenfors and Williams (2001). See in addition Gärdenfors (2000). 
Every point lying on the boundary of two or more terms is considered a borderline case of these predicates. The tessellation made by vague prototypes is presented in fig. 3.

\subsection{The ANS in the conceptual spaces framework}

Our central endeavor in this paper is to define a conceptual space that models a structure of ANRs related to the ANS. This system of ANRs is activated by discrete quantitative inputs. It is magnitude sensitive; that is, the bigger the quantity of the input, the bigger the magnitude represented in the system. ${ }^{5}$ The boundaries between elements of the ANS are not sharp: it is not clear whether 39 belongs to the scope of 45 , or whether this scope stops at 40. Moreover, the analogue magnitude representations obey the so-called Weber's Law: "the ability to discriminate two magnitudes is determined by their ratio. As the ratio of two magnitudes approaches 1:1 they become harder to discriminate, and beyond a certain threshold determined by the subject's 'Weber constant' cannot be discriminated at all” (Beck, 2014). On the neural level, a ratio 1:1 of two magnitudes in a noisy system makes it almost impossible to discriminate the neural entities. This effect of ratio-sensitivity occurs every time human adults have to discriminate magnitude when explicit counting is not possible (Barth et. al., 2003).

In this paper we target a particular type of AMRs' system. Therefore, there are multiple general aspects of such systems that we shall ignore in the proposed model. For example, there are various AMRs that are activated through different input - which underlies important differences, and gives rise to essentially different models, even though these AMRs share multiple common features and traits of functioning. Moreover, even the system of AMRs related to quantities is activated by input of various types (visual, aural, kinetic, or tactile). In this paper we want to avoid discussing the common nature of AMRs and the extent to which various inputs that activate ANS-related representations differ across types of AMR systems. We focus on AMRs that are activated in response to constant visual input consisting of various sets composed of different quantities of identical blue dots evenly distributed on a whiteboard. Even if the targeted objective of our research is to understand semantics for exact numerals, we will model the

${ }^{5}$ It is shown that analogue magnitudes related to the ANS get bigger in logarithmic progression (Dehaene, 2008; Berteletti et al., 2010). 
non-conceptual content of ANS-related representations, which occurs in this situation automatically before any other type of representation supporting quantitative information. Exact numerals and sensory quantitative, discrete input are claimed to activate the same system of representations (the ANS-related system of AMRs), so starting by proposing a sketch of a model for a simple sensory input seems to be a natural step to take.

The discussion of how exactly analogue magnitude representations are instantiated in the brain (are larger quantities represented by more neurons' firings or by faster firing of a fixed population of neurons?) does not fall within the scope of this paper. What is important for us is that "their psychophysical signatures strongly suggest analogue type of representational scheme" (Carey, 2009: 458). One simple way of thinking about this kind of representation is proposed by Carey and Sarnecka (2006: 477) and Carey (2009: 118): "[there exists] a helpful analogy to the following external system of analogue number representations. [...] Line length is a direct analogue of number. [... Suppose our brains deploy magnitude representations that are likewise analogue". Note that we do not take a position in the debate about how representations really look - that is irrelevant to our endeavor.

\author{
o o o \\ 00000 \\ 000000 \\ 00000000
}

Fig. 4. The representation of quantities in the ANS

Three

Five

Six

Eight

Fig. 5. Representation of numbers in the ANS 
The authors' original example focuses on symbolic input (fig. 5).

Indeed, it is claimed that when an agent acquires names for numbers, her neural reaction and activation of ANS-related representations will also take place when input is symbolic. Observe that the point we are making inscribes this line of reasoning in an even more straightforward way: our aim is to provide a model for vague-exact quantifiers.

We do not take up this challenge here, but it seems to us that there is a natural way of generalizing our model to other analogue magnitude representation systems. As put by Carey and Sarnecka (2006: 477),

$[\mathrm{n}]$ umber is not the only dimension of experience represented by analogue magnitudes other examples include brightness, loudness, and temporal duration. In each case, as the physical magnitudes get bigger, it becomes increasingly harder to discriminate values that are the same absolute distance apart. $[\ldots][\mathrm{T}]$ he discriminability of any two values is a function of their ratio $[\ldots]$.

\subsection{The ANS and prototypicality}

According to prototype theory, the cognitive process of categorization has a graded nature, i.e. some members of a category are better, more straightforward, or more central representatives of this category than other members (Rosch, 1973; Lakoff, 1987; Taylor, 2003). There exists serious empirical evidence confirming the prototype effect in human cognitive processes. One of the most famous series of experiments is Brent Berlin and Paul Kay's study of color categorization (Berlin, Kay, 1969). In the first series, respondents (competent users of different languages) were asked to divide a color continuum according to system of color categories functioning in their language. The results showed that ranges of color categories differ considerably from one language to the next. In a second series of experiments, subjects were asked to point out the best copy of a color among over three hundred color plates. Regardless of the different ranges of categories in their languages and their linguistic area, all participants pointed to the same color, i.e. same shade of, for instance, red. The results of Berlin and Kay's study show that even if languages categorize colors in different ways, for every color there exists a prototypical example, in common and similar across all languages.

As we said above, the ANS-related AMRs system represents the numerosity of a set by an analogue extension. Remember that in this paper we concentrate 
on a specific type of input, that is, we focus on AMRs that are activated in response to constant visual input consisting of various sets, which are composed of different quantities of identical blue dots distributed on a whiteboard in such a way that every dot is not further from some other dot than its diameter. For the moment we assume that dots can be organized in different shapes (spatially ordered or randomly distributed). Later, for the sake of model simplification, we will consider only these shapes, which are representable in the Cartesian coordinate system.

We argue that certain types of distribution of visual input are cognitively privileged. They activate ANS-related AMRs in the same way as the exact symbolic numerals do. This provides an opportunity to incorporate the phenomenon of prototypicality for model of ANS-related representations in the conceptual spaces framework. The distinctiveness of distribution can be studied, as we do it in this paper, from theoretical perspective, but we agree that the most significant results might be achieved through empirical investigation. Simply speaking, it is a plausible hypothesis that some shapes may be psychologically prototypical cases. In other words, in analogy to Berlin and Kay's color categorization experiment, some arrangements of blue dots on a board may be considered prototypical or more salient instances of a given cardinality than others.

Hypotheses presented in this paper could be tested empirically in experiments inspired by Berlin and Kay's work. One of our ambitions is to prepare theoretical and conceptual frameworks for such studies. To begin with, let us consider a two-dimensional conceptual space - with height and length as quality dimensions - in which we consider spatial arrangements of identical blue dots evenly distributed on a whiteboard. According to Berlin and Kay's result for color-predicates, there exists a language-independent set of prototypes. Subjects from the study converged on some unified set of examples when asked "what is a prototypical red?" Following Berlin and Kay, we offer the hypothesis that some of these arrangements will be more easily recognized by the subjects (competent users of the given language) as corresponding to a given numeral. The situation we want to describe is the following: subjects look at different quantities of identical dots. These dots can be arranged in different ways. The following figures contain just 4 dots, but this can be generalized to greater quantities. Moreover, as we said above, in the model construction we consider only spatially ordered shapes. ${ }^{6}$

${ }^{6}$ We are aware of limitations that this constraint entails and of the necessity of adjusting the proposed model to empirical results on prototypically. 

$\mathrm{O}$
$\mathrm{O}$
O O
$\mathrm{O} O \mathrm{O}$
$\mathrm{O} \quad \mathrm{O} O$
O O
$\mathrm{O}$
$\mathrm{O}$
0000
$\mathrm{O} \quad \mathrm{O} O$
$\mathrm{O}$

Fig. 6. Examples of arrangements of the four-element sets of dots in two-dimensional space

Similarly to the fig. 6, the extended, continuous, and approximate representations of the ANS also can be distinguished in terms of the space occupied by their shape:

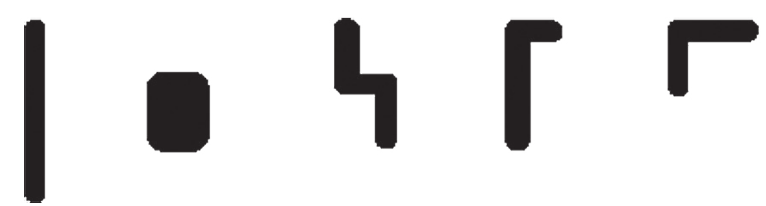

Fig. 7. The arrangements of shapes of 4-element sets of dots

Each of these possible arrangements varies according to height and length parameters and as such, they can be straightforwardly instantiated in conceptual spaces as ANS-related AMRs for "four". The hypothesis is the following: as in the case of colors, it seems pretty plausible that humans converge to some unified set of prototypes. Before an empirical study is conducted and a set of distribution recognized as prototypes psychologically grounded, we propose that in our model the role of the prototype is played by dots which are the most evenly distributed. ${ }^{7}$ The rest of the configurations are, in the proposed model, ordered following the degree of similarity to the prototype or prototypes.

To support our hypothesis that evenly distributed dots are more prototypical, one can refer to Tversky's studies provided in „Features of similarity” (Tversky, 1977). In two-dimensional conceptual space (height/length), the element of equal dimension ratio 1:1 is certainly a natural candidate for a prototype. The choice of an equilateral shape representation for the prototype is supported by Tversky's studies devoted to determining the degree of prominence of geometric

7 Again, it is just a simplification necessary for initiation discussion on conceptual spaces models for ANS-related representations. We use "evenly" with an undefined meaning, but we are aware that more exact definition is necessary. 
figures (Tversky, 1977). ${ }^{8}$ As Tversky puts it: „A major determinant of the salience of the geometric figures is goodness of form. Thus, a 'good figure' is likely to be more salient than a 'bad figure', although the latter is generally more complex" (ibid.: 334). Generally the more prominent or salient figure, the more regular and symmetric shape it has. The example of good and bad in form figures, given by Tversky, is presented on following figure:
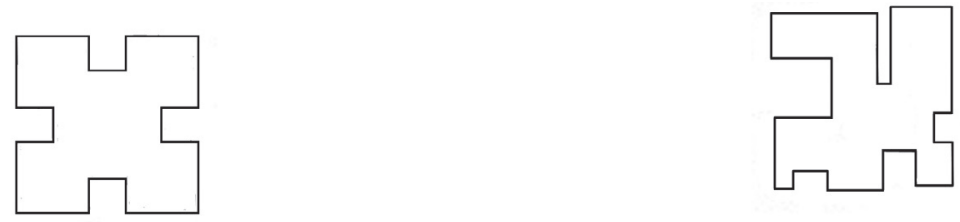

Fig. 8. More salient figure (left), and less salient one (right) (Tversky, 1977: 334)

For the sake of model construction, we propose to order the rest of the, configurations of 4-element sets of dots (mentioned above), following the degree of similarity to the prototype or prototypes. In other worlds the location of the other elements of the set is then determined by the degree of similarity to the prototype with regard to one of the dimensions, i.e. length and height. This is shown in the following figure:

\section{E N G T H}

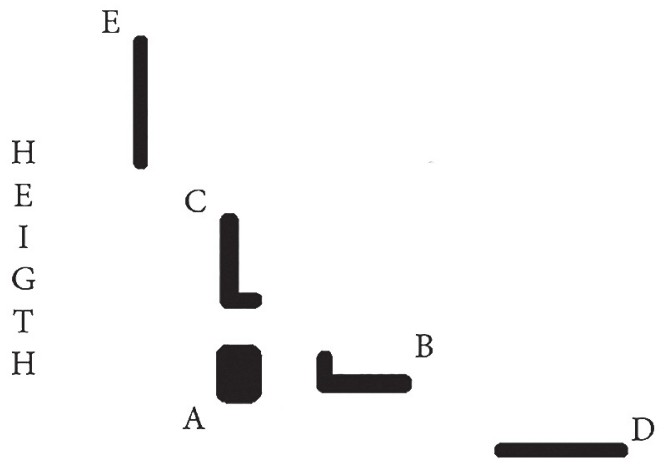

Fig. 9. Example of an ordered set of the arrangements of 4-element sets

${ }^{8}$ It can be argued that dots distributed in straight lines are in reality prototypical as well. In this paper we will not investigate this possibility. 
Element marked as A represents the prototype, the element marked as B differs from the prototype with regard of length dimension by 1 point on similarity scale. The element $\mathrm{C}$ also differs from the prototype by 1 on the similarity scale, but with regard of height dimension. $\mathrm{D}$ and $\mathrm{E}$ differ from prototype by 2 points with regard of length and height dimension respectively.

In the similar way we can model the sets of ANS-related representations of other magnitudes. Models for bigger numbers will differ from the above, because these representations take into their scope neighboring cardinalities. So, for example, some inputs that activate the representation of 17 also activate some (non prototypical) representations of 16 and 18 . The number of representations taken into account by the AMR for each number grows with magnitudes of elements from the number line.

The application of Voronoi tessellation to the conceptual spaces model allows us to map the entire conceptual representation structure of size provided by the ANS. The set of generators of the tessellation will be constituted by the collection of prototypes of each subsequent quantity represented by ANS magnitudes (i.e. prototypes of $4,5,6$, and so on). Therefore, if we treat points as representations of successive prototypes of numeric quantities, we will get the following picture of the conceptual structure of the ANS.

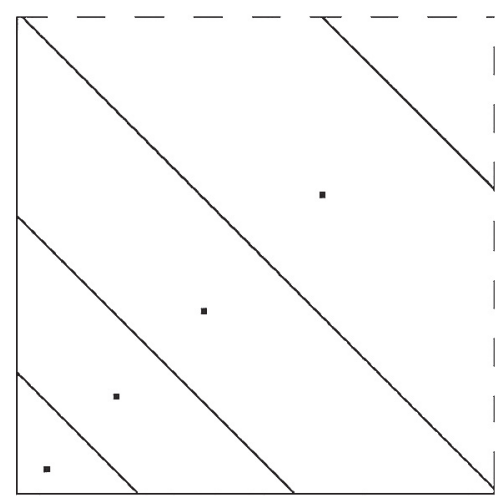

Fig. 10. The idealized conceptual structure of the ANS

However, the above-presented conceptual structure of ANS representations is certainly not vague. It should be noted that modeling the approximate nature of ANS-related representations involves taking into account the blurred borders in the above scheme. This objective can be achieved by using the fuzzy prototype 
model (the so-called prototype groups) proposed by (Douven et al., 2013). In short, this model requires treating cases of objects adjacent to the prototype also as prototypical cases, and using them as generators of subsequent tessellations. As a result, we get the following diagram:

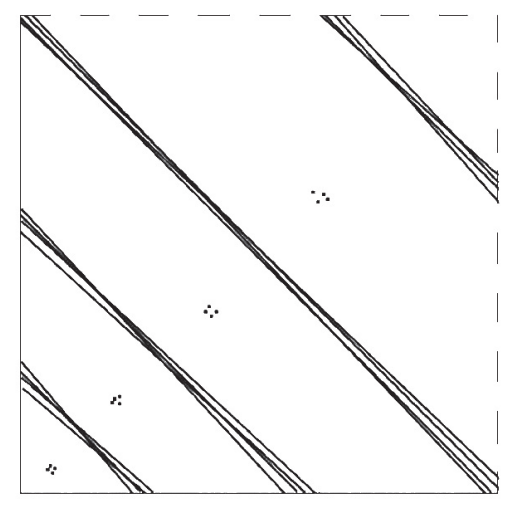

Fig. 11. The conceptual spaces structure of the ANS

Thicker boundaries of tessellation model boundary cases where the number of elements in the set might or might not activate the representation which are activated by prototypes. Figures 9 and 10 contain a preliminary visualization of the proposed model. We suspect that for larger numerical values regions of tessellation will differ from the picture above. Regions representing larger numerical values are not only contiguous upon the regions representing two closest values in numeral progression, but also are contiguous upon other proximate regions. We say that region $A$ is contiguous upon the region $B$, iff a ratio of numerical values represented by both regions is close as possible to $1: 1$. The exact value of the ratio should be ascertained experimentally, but for the purposes of the model, we can venture to say that the deviation should not exceed 0.1 . This rule explains why the region representing 5 in conceptual space is contiguous only upon regions denoting 4 and 6 , since the ratio between the 5 and 4 and between 5 and 6 is respectively 1.25 (deviation $=0.25)$ and 0.83 (deviation $=0.23)$. While the region representing for instance 33 is contiguous upon 6 regions (i.e. representing $30,31,32,34,35,36)$, since deviation of a ratio between 30 and other numbers is smaller than 0.1 .

Understanding the contiguousness of regions in terms of the ratio of values representing by them is the consequence of the characteristic for ANS size and 
distance effects. According to the latter it is easier to discriminate - with respect to a given set - a set that contains more elements than a set with a similar number of elements to the comparison set. In other words, if one compares two numbers to the same target, the number that is further away from the target should be easier to discriminate. For instance, it is easier to tell the difference between 10 and 15 than between 10 and 11 . Concepts arranged close to each other in conceptual space, since they have a common vague area (i.e. a fuzzy boundary), are much more difficult to distinguish than concepts arranged further away from each other in space.

According to the size effect, when the absolute difference between the two pairs of sets is constant, it is easier to compare sets with a lower number of elements. This effect can be quite intuitively achieved in the presented model. As a matter of fact, the size effect occurs simply due to the way that the magnitude of representation is modeled in conceptual space. This is because in the proposed model, the magnitude of the representation binds directly with the amount of variants of the objects' spatial orientations. Both values (i.e. magnitude of the representation and amount of variants of the objects' spatial orientations) have a direct impact on the size of representation and the number of prototypes, and as a consequence also on the size of the prototype group. The size of the prototype group has a direct bearing on the size of the penumbra - i.e. the range of the blurred boundary between adjacent terms in the series. In other words, the larger the sets that are compared, the easier it is to make a mistake, since the absolute difference between sets is small enough to be located within the penumbra area.

It is important to note that the proposed model of the conceptual framework of ANS-related representations is easily and intuitively reconciled with the ANS's distance and size effects. Both effects show that similarity between ANS-related representations in conceptual space is consistent with Weber's law. This means that the degree of similarity is designated by the inverse ratio of two magnitudes being compared with each other. In other words, the smaller the ratio between the magnitudes, the more similar objects are.

\section{Conclusions and openings}

In this paper we discussed the ANS-related system of representations. These representations firstly get activated by preverbal, empirical input, and then continue to be activated when symbols from number line acquire meaning. The 
model based on conceptual spaces that we proposed, aims at accounting for the structure of these representations. In consequence, additional light is shed on the relation between exact numerals, especially when they are used as vague quantifiers, ANS-related representations as a (naturalized) semantics for such expressions and a model of the ANS-related representations in the framework of conceptual spaces.

The model we propose is based on the idea that tessellations corresponding to ANS-related representations are generated not by a single prototype, but by clusters of prototypes. In consequence boarders between concepts are fuzzy. We argue that this model accounts for size and distance effects, characteristic for ANS.

We believe that further investigation into the exact structure of this model is necessary, in particular necessary is to take into account experimental data disclosing sets of empirical inputs, which correspond to prototypical representations. Another interesting opening, which we do not explore in this paper, is the relation between activation of the system of analog magnitude representations by non-symbolic input and activation of this system by symbolic input (language, number names, numerals). Such a study would enhance understanding of the semantic of numerals.

\section{References}

Beck, J. (2014). Analogue magnitude representations: A philosophical introduction, British Society for the Philosophy of Science 66 (4): 829-855.

Barth, H., Kanwisher, N., Spelke, E. (2003). The construction of large number representations in adults. Cognition 86: 201-221.

Berlin, B. Kay, P. (1969). Basic Color Terms: Their Universality and Evolution, Berkeley: University of California Press.

Berteletti, I., Lucangeli, D., Piazza, M., Dehaene, S., Zorzi, M., (2010). Numerical estimation in preschoolers, Developmental Psychology 46 (2): 545-551.

Carey, S., (2001). Cognitive foundations of arithmetic: Evolution and ontogenesis. Mind and Language 16: 37-55.

Carey, S., (2009). The Origin of Concepts. New York: Oxford University Press.

Carey, S., Sarnecka, B.W. (2006). The development of human conceptual representations. In: M. Johnson, Y. Munakata (eds.), Processes of Change in Brain and Cognitive Development: Attention and Performance XXI (pp. 473-496). New York: Oxford University Press.

Decock, L., Douven, I. (2014). What is graded membership? Noûs 48: 653-682.

Dehaene, S. (1997). The Number Sense. New York: Oxford University Press.

Dehaene, S. (2008). Symbols and quantities in parietal cortex: Elements of a mathematical theory of number representation and manipulation. In: P. Haggard, Y. Rosetti,

\section{6 \&) COgNitive sCIEnCE}


M. Kawato (eds.), Sensorimotor Foundations of Higher Cognition (pp. 527-574). New York: Oxford University Press.

Douven, I., Decock, L., Dietz, R., Égré, P. (2013). Vagueness: A conceptual spaces approach, Journal of Philosophical Logic 42 (1): 137-160.

Feigenson, L., Dehaene, S., Spelke, E.S. (2004). Core systems of number. Trends in Cognitive Sciences 8 (7): 307-314.

Gallistel, Ch. R. (1993). The Organization of Learning, Cambridge MA: MIT Press.

Gärdenfors, P. (2000). Conceptual Spaces, Cambridge MA: MIT Press.

Gärdenfors, P., Williams M.-A. (2001). Reasoning about Categories in Conceptual Spaces. In: Proceedings of the Fourteenth International Conference of Artificial Intelligence (pp. 385-392), San Francisco CA: Morgan Kaufmann.

Halberda, J., Feigenson, L. (2008). Developmental change in the acuity of the 'Number Sense': The approximate number system in 3-, 4-, 5-, 6-year olds and adults. Developmental Psychology 44: 1457-1465.

Krifka, M. (2009). Approximate interpretations of number words: A case for strategic communication. In: E. Hinrichs, J. Nerbonne (eds.), Theory and Evidence in Semantics (pp. 109-132). Stanford: CSLI Publications.

Lakoff, G. (1987). Women, fire and dangerous things: What categories reveal about the mind, Chicago: University of Chicago Press.

McGee, V., McLaughlin, B. (1994). Distinctions without a difference, Southern Journal of Philosophy 33 (suppl.): 203-251.

Mussolin, C., Nys, J., Leybaert, J., Content, A. (2012). Relationships between approximate number system acuity and early symbolic number abilities. Trends in Neuroscience and Education 1: 21-31.

Nagen, J., Sarnecka, B. (2015), Is there really a link between exact-number knowledge and approximate number system acuity in young children? British Journal of Developmental Psychology 33 (1): 92-105.

Rosch, E.H. (1973) Natural categories, Cognitive Psychology 4: 328-350.

Shapiro, S. (2006). Vagueness in Context. New York: Oxford University Press.

Solt, S. (2011). Vagueness in quantity: Two case studies from a linguistic perspective. In: P. Cintula, Ch. G. Fermüller, L. Godo, P. Hajek (eds.), Understanding Vagueness. Logical, Philosophical and Linguistic Perspectives (pp. 157-174). London: College Publications.

Spelke, E.S., Kinzler, K.D. (2007). Core knowledge. Developmental Science, 10: 89-96. Taylor, J.R. (2003). Linguistic Categorization, New York: Oxford University Press.

Tversky, A. (1977). Features of similarity. Psychological Reviews 84 (4): 327-352.

Wagner, J.B., Johnson, S.C. (2011). An association between understanding cardinality and analog magnitude representations in preschoolers. Cognition 119: 10-22. 